\title{
Adjustment of foreign EQ-5D-3L utilities can increase their transferability
}

This article was published in the following Dove Press journal:

ClinicoEconomics and Outcomes Research

15 December 2015

Number of times this article has been viewed

\author{
Lars Oddershede ${ }^{1,2}$ \\ Karin Dam Petersen' \\ 'Danish Center for Healthcare \\ Improvements, Faculty of Social \\ Sciences, Faculty of Health Sciences, \\ Aalborg University, Aalborg East, \\ ${ }^{2} \mathrm{HEOR}$ Consult ApS, Copenhagen, \\ Denmark
}

Background: Foreign utilities of the EQ-5D-3L (3-level version of the EuroQol-5 Dimension of health questionnaire) are not readily transferrable to economic evaluations conducted from a national perspective. It has been advised to avoid transferring mean utilities from one country to another without adjusting them; yet no such method exists.

Purpose: The present study aimed to develop a method for adjusting mean utilities to increase their transferability from one country to another.

Methods: Seven datasets containing EQ-5D-3L answers were valued using value sets from four countries: the UK, the Netherlands, Germany, and Spain. Hereby, seven mean utility values were obtained for each country. This allowed for three pairwise comparisons: 1) UK mean values vs Dutch mean values; 2) UK mean values vs German mean values; and 3) UK mean values vs Spanish mean values. For each of these three comparisons, a regression model was fitted using the mean UK utilities as the dependent variable and the other country's mean utilities as the independent variable. The coefficients from the three regression models were validated using results from a published article containing mean utilities obtained by valuing the EQ-5D-3L data using all four value sets.

Results: The findings suggested that adjustment of foreign utilities may increase transferability between countries where value sets are not comparable. It was possible to adjust the mean utilities valued by the Dutch and German value sets to make them reflect mean UK utilities as there were substantial differences between these value sets. Transferability of the Spanish mean utility values was not improved as the Spanish and UK value sets are sufficiently similar.

Conclusion: It is feasible to adjust foreign mean utilities of the EQ-5D-3L to make them reflect national preferences for health.

Keywords: QALY, generalizability, health-related quality of life, national preference weights, value set, tariff

\section{Introduction}

Markov modeling is frequently used to estimate the costs and consequences of two or more interventions for a specific condition. ${ }^{1}$ In these models, the consequence of each treatment is often estimated in terms of quality-adjusted life-years (QALYs). In order to calculate the QALYs accrued, the life years are weighted by the quality of life, also referred to as the utility. In the UK, utility estimates should preferably be obtained by valuing the answers of the generic EuroQol-5 Dimension (EQ-5D) questionnaire using the UK value set. ${ }^{2}$ However, analysts may be unable to obtain EQ-5D utilities valued by the national value set and therefore be forced to use foreign EQ-5D utilities. ${ }^{3}$ Furthermore, evidence shows that foreign utilities of the EQ-5D-3L are not transferable to another country because of differences in living standards, health care system
Correspondence: Lars Oddershede Danish Center for Healthcare Improvements, Faculty of Social Sciences, Faculty of Health Sciences, Aalborg University, Fibigerstraede II room 70, 9220 Aalborg East, Denmark Tel +45 40609448

Email lars.oddershede@gmail.com 
performance, and cultural differences between countries. Cross-country differences are due to a mixture of differences in: 1) how people in each country report problems along the five dimensions, ${ }^{4}$ 2) how different populations value these problems, ${ }^{5}$ and 3 ) how the value sets are elicited and modeled. ${ }^{6}$ Thus, the choice of value set will consequently influence the cost-effectiveness results. ${ }^{5,7-15}$ Therefore, analysts are advised to adjust foreign utilities of the EQ-5D, before applying them in an economic evaluation conducted from a national perspective. ${ }^{7}$ In a worst-case scenario, application of unadjusted utilities may lead to biased cost-effectiveness results and wrong decision-making. Although adjustment of foreign utilities is advised, no such method has been developed.

The present study aimed to develop a method for adjusting mean utilities of the 3 level version of the EQ-5D (EQ-5D-3L) to increase their transferability from one country to another.

If foreign mean utilities of the EQ-5D-3L identified through a literature review could be adjusted to increase their transferability, more utility data could be considered relevant and pooled. This would improve precision in the estimated mean utility and hence improve accuracy and precision of the corresponding cost-effectiveness result. ${ }^{3}$

\section{Methods}

\section{Outline of the analysis}

The study investigated if it was possible to develop a simple mathematical model, ie, a simple equation, which could be used to adjust a foreign mean utility, valued by either the Dutch, German, or Spanish value sets, to make it reflect UK preferences for health. If a Dutch, German, and Spanish mean utilities could be adjusted to reflect UK preference for health, it would increase the foreign utilities transferability to a UK setting. Although four value sets from specific countries are used to develop a method for adjusting mean utilities to increase their transferability from one country to another, the countries merely serve as an example. The method could have been developed using the value sets from any country.

Seven datasets containing EQ-5D-3L answers were used to develop methods for adjusting foreign utilities to increase their transferability. The data used will be discussed later in the paper. The EQ-5D-3L answers in each dataset were then valued using four different time trade-off value sets. As such, four mean utilities were obtained from each dataset using the value sets from: the UK, ${ }^{16}$ the Netherlands, ${ }^{17}$ Germany, ${ }^{18}$ and Spain. ${ }^{10}$ This allowed for three pairwise comparisons of: 1) UK mean values vs Dutch mean values; 2) UK mean values vs German mean values; and 3) UK mean values vs Spanish mean values. For each of these three comparisons, a regression model was fitted using the mean UK utilities as the dependent variable and the other country's mean utilities as the independent variable. The coefficient from these three regression models enabled to adjust: 1) Spanish mean utilities to UK mean utilities; 2) German mean utilities to UK mean utilities; and 3) Dutch mean utilities to UK mean utilities. To investigate the performance of the method for adjusting mean utilities from one country to another, the method was validated on an external dataset. ${ }^{9}$

\section{Choice of value sets}

The four value sets were chosen to illustrate that utilities can be adjusted to increase their transferability for two reasons. The first reason is that this choice enabled external validation of the method. A previously published study applied the same four value sets to their EQ-5D-3L responses in a study of acute cough/lower respiratory tract infections at five timepoints. ${ }^{9}$ These five mean utilities for each of the four value sets could be used as an external dataset in which the three equations for adjusting mean utilities could be validated. The second is that the choice enabled the assessment of whether adjustment increases transferability when value sets are comparable (Figure 1A; the Spanish and the UK value set), and whether adjustment is feasible when the value sets differ at both high and low values (Figure 1B; the German and the UK value set), and if adjustment is feasible when high values are comparable between value sets but low values are not (Figure 1C; the Dutch and the UK value set).

\section{Data sources used to estimate the equations for adjusting mean utilities}

Seven datasets containing EQ-5D-3L answers from six studies were used to generate an estimation dataset containing seven mean utility values from each of the four countries. ${ }^{19-24}$ The studies included a trial which included patients with human immunodeficiency virus, ${ }^{20}$ a representative sample of the adult population in the North Denmark Region, ${ }^{23}$ a trial comparing case-management to current treatment for patients suffering from chronic obstructive pulmonary disease, ${ }^{24}$ a trial comparing a telehealthcare intervention to current treatment for chronic obstructive pulmonary disease patients, ${ }^{21}$ a mapping study within cardiovascular disease, ${ }^{19}$ and a trial investigating allergy patients quality of life on days with and without symptoms, hereby providing two mean values for the estimation dataset. ${ }^{22}$ From each of these studies, descriptive tables of frequencies of observed EQ-5D-3L health states were kept and this made it 

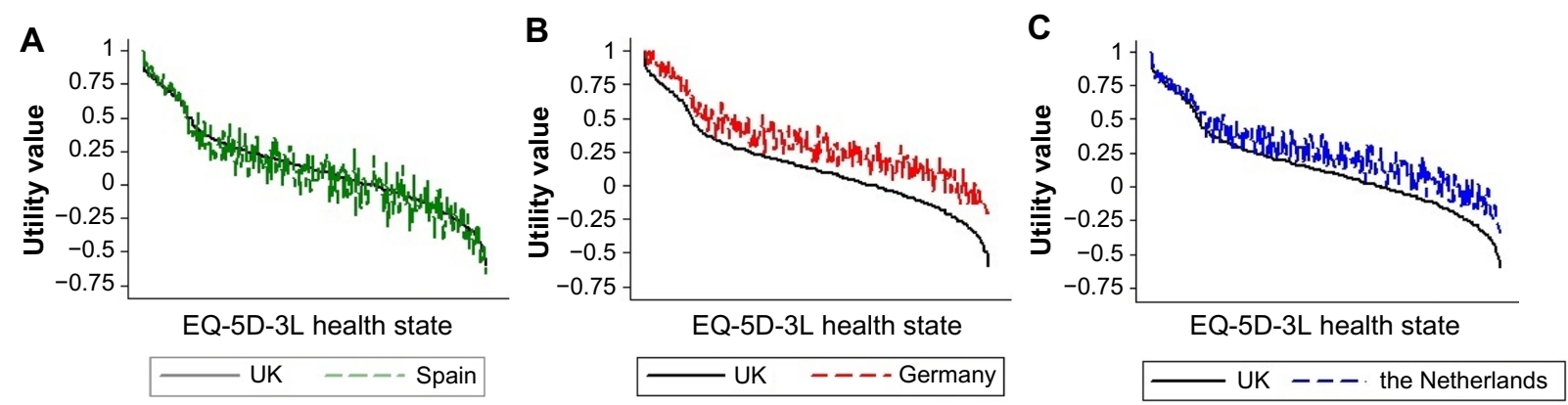

Figure I Pairwise comparison of the four value sets used in the present study.

Notes: The graph shows (A) the utility values which can be obtained when applying the value sets from the UK and Spain to the 243 health states of the EQ-5D-3L questionnaire, (B) the utility values which can be obtained when applying the value sets from the UK and Germany, and (C) the utility values which can be obtained when applying the value sets from the UK and the Netherlands. Health states are ordered so that the $\mathrm{x}$-axis runs from the least severe health state in the UK value set to the most severe health state in the UK value set.

Abbreviation: EQ-5D-3L, 3-level version of the EuroQol-5 Dimension of health questionnaire.

possible to attach the UK value set and the three foreign value sets to the same data. Hereby, seven mean utility values were obtained from each of the four value sets ${ }^{10,16-18}$ (Table 1).

\section{Estimating the equations for adjusting mean utilities}

Three regression models were estimated using the UK mean utility as the dependent variable and either the Dutch, the German, or the Spanish mean utility as the independent variable. This was done to predict: 1) UK mean utility values from Dutch mean utilities; 2) UK mean utility values from German mean utilities; and 3) UK mean utility values from Spanish mean utilities. Ordinary least-squares regression models were used to estimate all three models. It was deliberately chosen to restrict the functional form for the regression to simple linear regression. In many valuation studies, an N3 term is included to capture the known interaction effect. Although the interaction effects must be tended to in valuation studies, it is not self-evident that there are any such relationships between mean utilities from two countries. The relationship may be a simple linear relationship. Parsimonious models were therefore chosen as the aim of the present study to show that methods for adjusting mean utilities can be developed. Formally, the regression model for predicting a mean UK utility value from a Dutch mean utility value could be written as

$$
\text { Utility }_{\mathrm{j}}^{\mathrm{UK}}=\beta_{0}+\beta_{1} \cdot \text { Utility }_{\mathrm{j}}^{\mathrm{NL}}
$$

where the index $j$ identifies the dataset $(j=1,2 \ldots, N)$, Utility $_{\mathrm{j}}{ }^{\mathrm{UK}}$ and $\mathrm{Utility}_{j}^{\mathrm{NL}}$ are the mean utilities of the EQ-5D-3L valued by the UK and the Dutch value set, respectively. The coefficient $\beta_{0}$ is the expected mean UK utility when the mean Dutch utility equals zero. The coefficient $\beta_{1}$ is the marginal increase in the mean UK utility at increasing values of mean Dutch utility. It was chosen to present the root mean squared error, the mean absolute error, and the $R$-squared for each of the regression models for descriptive purposes. All regression models were generated in Stata version 13.1 (StataCorp LP, College Station, TX, USA).

\section{Data source used to validate the equations for adjusting mean utilities}

In a study involving 1,327 patients with acute cough/lower respiratory tract infection, EQ-5D-3L data were collected in the Netherlands, Germany, Spain, and four other countries at five time-points. The EQ-5D-3L data from the Netherlands,

Table I Data used to develop the equations for adjusting utilities

\begin{tabular}{|c|c|c|c|c|}
\hline \multirow[t]{2}{*}{ Study } & \multicolumn{4}{|c|}{ Mean utility obtained using the value set from } \\
\hline & United Kingdom & the Netherlands & Germany & Spain \\
\hline General population ${ }^{23}$ & 0.8299 & 0.8547 & 0.8963 & 0.8619 \\
\hline Human immunodeficiency virus ${ }^{20}$ & 0.8525 & 0.8606 & 0.9206 & 0.8827 \\
\hline Cardiovascular disease $\mathrm{e}^{19}$ & 0.6460 & 0.7070 & 0.7664 & 0.6537 \\
\hline Case-management in chronic obstructive pulmonary disease $\mathrm{e}^{24}$ & 0.6457 & 0.6916 & 0.7710 & 0.6607 \\
\hline Telehealthcare in chronic obstructive pulmonary disease ${ }^{21}$ & 0.6478 & 0.7031 & 0.7666 & 0.6571 \\
\hline Allergy patients on a day without symptoms ${ }^{22}$ & 0.9778 & 0.9794 & 0.9917 & 0.9866 \\
\hline Allergy patients on a day with symptoms ${ }^{22}$ & 0.7324 & $0.779 \mid$ & 0.8329 & $0.764 I$ \\
\hline
\end{tabular}


Germany, and Spain were valued both with the country's value set and the UK value set (Table 2 ).

These results were used to assess the performance of the three equations on external data. This was done by drawing two Bland-Altman plots per equation. The first was drawn to compare the agreement between the observed foreign mean utilities and the observed UK mean utilities. The second was drawn to compare the agreement between the adjusted foreign mean utilities and the observed UK mean utilities. Using these pairwise plots, it was possible to assess if there was better agreement between the observed mean UK utility and the adjusted foreign mean utility than there was between the observed mean UK utility and the observed foreign mean utility.

\section{Results}

\section{Estimation}

Three regression models were estimated to obtain three equations for adjusting mean utilities and the results are summarized in Table 3.

In general, the foreign mean utility values were highly correlated to the mean UK utility values. $R$-squared values of all three regression models were $>0.99$. The coefficients of the model predicting mean UK utility from the mean Spanish utility had a $\beta_{0}$ close to 0.0 and a $\beta_{1}$ close to 1.0. As such, the mean Spanish utilities were not systematically different from the mean UK utilities. This was not the case for the Dutch and the German mean utility. Both the Dutch and the German mean utility were systematically higher than the mean UK utilities as the $\beta_{0}$ in their regressions were below zero and the $\beta_{1}$ in was above 1.0.

\section{Validation}

Figure 2 shows the six Bland-Altman plots drawn to assess the equations ability to adjust foreign mean utilities to make the values more transferable and thereby applicable in economic evaluation performed from a UK perspective.

When comparing the agreement between the observed mean UK utilities and the adjusted mean Dutch utilities, Figure 2A, to the agreement between the observed mean UK utilities and the observed mean Dutch utilities, Figure 2B, it is seen that agreement was better when using the equation to adjust the foreign utilities, ie, Figure 2A. Likewise, the adjusted mean German utilities were most comparable to the observed mean UK utilities. However, this was not the case for the mean Spanish utilities. When applying the equation for adjusting mean Spanish utilities of the EQ5D-3L, a slight systematic bias was induced. As such, the observed mean Spanish utilities were directly transferable to the UK setting, whereas observed mean Dutch and German utilities benefitted from being adjusted, using the respective regression models.

\section{Discussion}

It is known that international utility values cannot blindly be transferred to a national setting as EQ-5D-3L responses and value sets may differ for multiple reasons. As such, utility values from foreign studies must be adjusted before applying them in economic evaluations. This study developed a method for adjusting mean utilities to make them transferable from one country to another. The method presented does not handle potential differences in EQ-5D-3L responses - it merely predicts what the mean utility would have been if another value set had been used.

In the present study, the case of adjusting utilities to UK preference for health was chosen. However, the principal could be applied to develop equations for adjusting utilities from any country to a particular country. Our results showed that the equations for adjusting utilities were successful in adjusting the Dutch and German utilities. This was evident

Table 2 Mean utilities used to validate the equations for adjusting mean utilities

\begin{tabular}{|c|c|c|c|c|c|}
\hline \multirow[t]{2}{*}{ EQ-5D-3L data for the pairwise comparison of } & \multicolumn{5}{|c|}{ Mean utility at } \\
\hline & Baseline & Week I & Week 2 & Week 3 & Week 4 \\
\hline \multicolumn{6}{|l|}{ UK and the Netherlands } \\
\hline UK value set applied to the EQ-5D-3L answers & 0.719 & 0.764 & 0.856 & 0.897 & 0.905 \\
\hline Dutch value set applied to the EQ-5D-3L answers & 0.768 & 0.807 & 0.883 & 0.915 & 0.922 \\
\hline \multicolumn{6}{|l|}{ UK and Germany } \\
\hline UK value set applied to the EQ-5D-3L answers & 0.713 & 0.788 & 0.882 & 0.920 & 0.930 \\
\hline German value set applied to the EQ-5D-3L answers & 0.825 & 0.870 & 0.934 & 0.958 & 0.962 \\
\hline \multicolumn{6}{|l|}{ UK and Spain } \\
\hline UK value set applied to the EQ-5D-3L answers & 0.721 & 0.823 & 0.912 & 0.935 & 0.938 \\
\hline Spanish value set applied to the EQ-5D-3L answers & 0.730 & 0.799 & 0.901 & 0.927 & 0.930 \\
\hline
\end{tabular}

Note: Data obtained from Oppong et al. ${ }^{9}$

Abbreviation: EQ-5D-3L, 3-level version of the EuroQol-5 Dimension of health questionnaire. 
Table 3 Equations for adjusting foreign mean utilities to UK utilities

\begin{tabular}{llll}
\hline Parameter & \multicolumn{2}{l}{ Ordinary least squares regression } & \\
\cline { 2 - 4 } & $\begin{array}{l}\text { Adjusting Dutch utilities } \\
\text { to the UK } \beta \text { (SE) }\end{array}$ & $\begin{array}{l}\text { Adjusting German utilities } \\
\text { to the UK } \beta \text { (SE) }\end{array}$ & $\begin{array}{l}\text { Adjusting Spanish utilities } \\
\text { to the UK } \beta \text { (SE) }\end{array}$ \\
\hline Foreign mean utility value & $1.2030^{* *}(0.0360)$ & $1.4475^{* *}(0.0379)$ & $0.9715^{* *}(0.0367)$ \\
Constant & $-0.1965^{*}(0.0289)$ & $-0.4678^{* *}(0.0324)$ & $0.0030(0.0290)$ \\
Estimation sample size, $\mathrm{n}=$ & 7 & 7 & 7 \\
R-squared & 0.9956 & 0.9966 & 0.9929 \\
Mean absolute error & 0.0068 & 0.0059 & 0.0090 \\
Root mean squared error & 0.0095 & 0.0083 & 0.0119 \\
\hline
\end{tabular}

Notes: $* P<0.01$; $* * P<0.001$.

Abbreviation: SE, standard error.
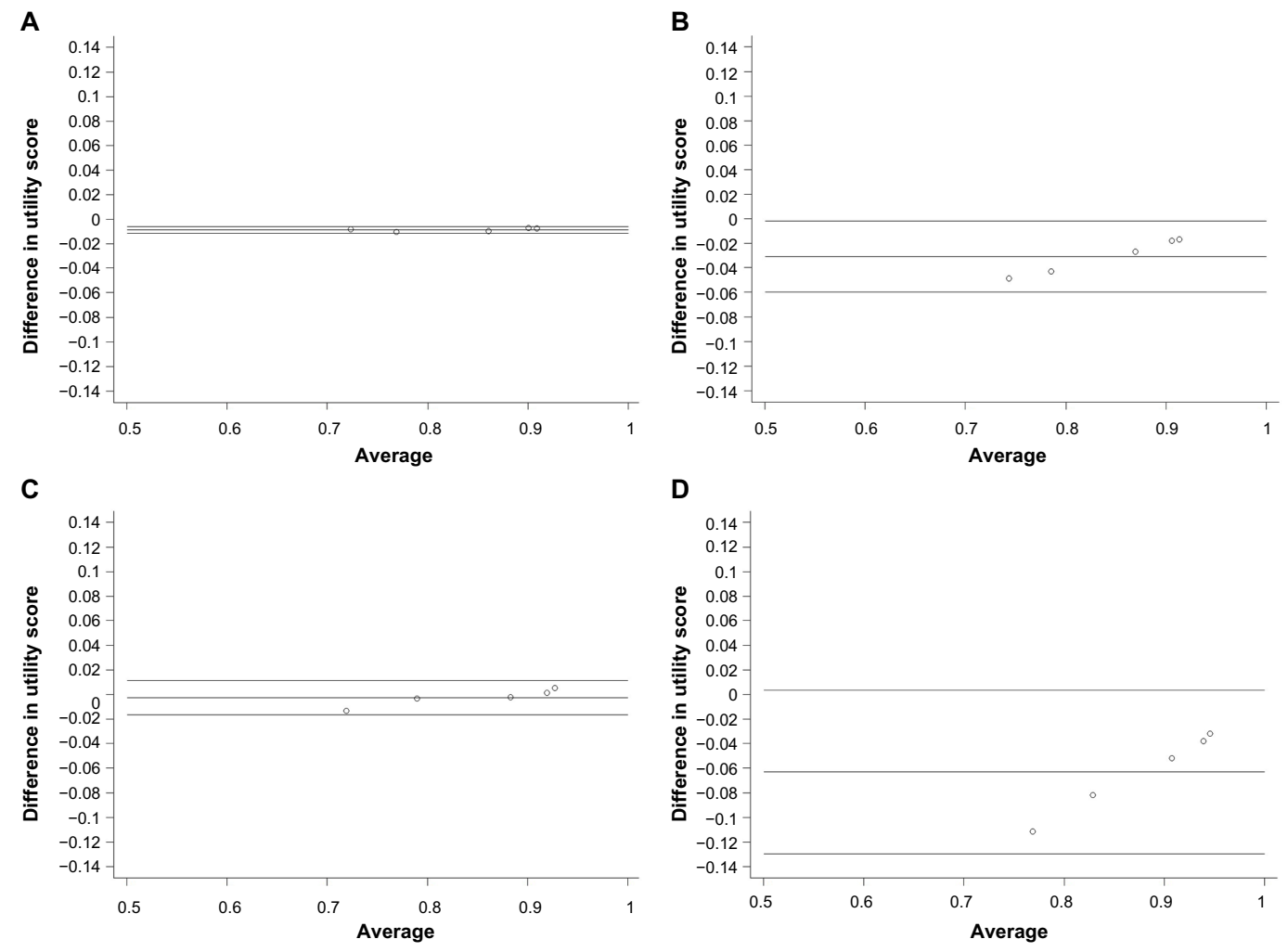

D
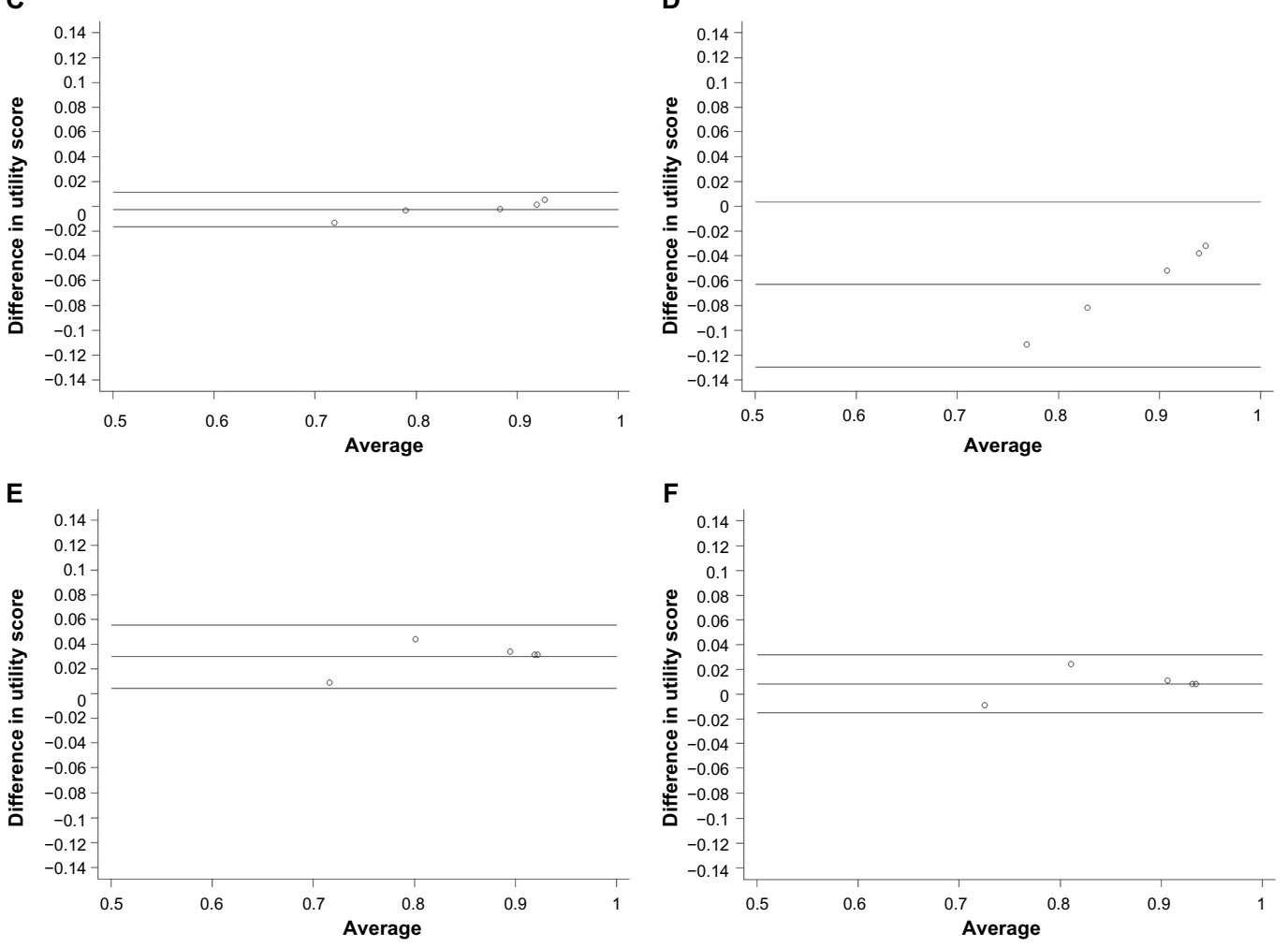

Figure 2 Bland-Altman plots of agreement.

Notes: Agreement between (A) the observed mean EQ-5D-3L UK utilities and the adjusted Dutch mean utilities, (B) the observed mean UK utilities and the observed Dutch mean utilities, (C) the observed mean UK utilities and the adjusted German mean utilities, (D) the observed mean UK utilities and the observed German mean utilities, (E) the observed mean UK utilities and the adjusted Spanish mean utilities, $(\mathbf{F})$ the observed mean UK utilities and the observed Spanish mean utilities. There is better agreement between observed mean UK utilities and adjusted Dutch and German utilities than between observed mean UK utilities and observed Dutch and German utilities. As such, adjustment improves transferability of Dutch and German utilities to a UK setting. Adjustment of Spanish mean utilities does not seem to improve transferability to a UK setting. Abbreviation: EQ-5D-3L, 3-level version of the EuroQol-5 Dimension of health questionnaire. 
as the adjusted Dutch and German mean utilities were closer to the observed UK mean utilities than the observed Dutch and German mean utilities were. As such, the adjustment of Dutch and German mean utilities made them transferable to the UK setting. It is important to note that the equation for adjusting mean utilities from a particular country to make them transferable to the UK may also work the other way around be rearranging it slightly. The equation for adjusting Dutch utilities to a UK setting can be rearranged to adjust UK utilities to a Dutch setting:

$$
\begin{aligned}
\text { Utility }^{\mathrm{UK}} & =1.2030 \cdot \text { Utility }^{\mathrm{NL}}-0.1965 \\
\uparrow & \text { Utility }^{\mathrm{NL}}=\left(\text { Utility }^{\mathrm{UK}}+0.1965\right) / 1.2030
\end{aligned}
$$

As such, it is only necessary to generate a single good equation for adjusting mean utilities between two countries. It is not certain that a simple linear relationship as the one presented in this paper is suitable for the entire range of mean utility values and further attention should be directed at this in future research. Likewise, this paper shows that foreign mean utilities may be adjusted to increase their transferability but it does not assess whether the error in the mean estimate, ie, the standard error, should also be adjusted. Furthermore, our results suggested that mean utilities obtained by valuing EQ-5D-3L answers using the Spanish value set are comparable to mean utilities obtained by valuing EQ-5D-3L answers using the UK value set. In other words, the Spanish utilities did not need to be adjusted to be transferable to the UK setting because the two value sets are very sufficiently comparable. However, a more formal rule for when adjustment is needed needs to be developed.

Many countries guidelines for economic evaluation have taken a position toward the transferability of utilities between countries. ${ }^{11}$ It appears that more recent versions of guidelines pay an increasing attention to the issues with the transferability of utilities and are more restrictive in their acceptance of data from other countries. The implication of the method presented in this study is that mean utilities of the EQ-5D-3L obtained from international studies could easily be adjusted to increase their transferability to a national setting.

By making more utilities identified from a literature review relevant to a decision analytic model be adjusted, the means for use of foreign value sets could potentially mean that more data could be pooled, hereby improving precision and providing better information for decision-makers. Failing to adjust mean utilities from international studies before using them in a national economic evaluation will lead to biased cost-effectiveness results, if there are country-specific differences in health perception.

International pharmaco-economic guidelines take different standpoints on the transferability of utility values. ${ }^{11}$ Two out of 27 guidelines consider utilities generalizable, three consider utilities transferable under extreme circumstances, three consider utilities transferable with adaptation, three do not consider utilities transferable, and the remaining 16 guidelines do not state an opinion on the matter. ${ }^{11}$ Like the majority, the Dutch, German, and Spanish guidelines do not state whether utilities can be considered transferable, while the UK guidelines do not consider utilities as transferable. Although the adjustment method presented in this paper is not sufficient to suggest that utilities should be considered transferable in pharmaco-economic guidelines, it can increase the transferability of utilities regardless of the local guideline's standpoint.

\section{Strengths and limitations}

The adjustment method presented in this study showed a promising ability to adjust a foreign mean utility, making the value transferable to the UK setting. The feasibility of developing such methods was strengthen by the validation of the method on external data which showed that the equations from the regression models were able to adjust the Dutch and German mean utilities to make them reflect the mean which would have been obtained if the EQ-5D-3L data had been valued with the UK value set.

However, the appropriateness of foreign EQ-5D-3L data in UK economic evaluations could be questioned. When an economic evaluation is informed by using EQ-5D-3L data collected in a foreign country and applying the national value set to the data, an important, often implicit, assumption is made. The assumption is that the distribution of EQ-5D-3L health states observed in particular severity of a given condition does not vary, ie, respondents in the two countries answer the EQ-5D-3L in sufficiently similar ways. In order to apply the method presented in this study, the same assumption applies. This assumption might be somewhat problematic as culture, country of origin, and income may impact the respondent's answers to the EQ-5D-3L. ${ }^{4}$ However, this assumption is often accepted by the National Institute for Health and Care Excellence (NICE) in the UK as descriptive EQ-5D data from multinational trials can be used to inform economic evaluations as long as the UK value set is applied. As such, the assumption that the distribution of EQ-5D-3L health states observed in particular severity of a given condition 
does not vary between the countries and should not hinder the pragmatic analyst in adjusting foreign mean utility values to make them reflect national preferences for health. There are, however, several other issues which should be addressed before this novel method can become a routinely used method for adjusting foreign utilities.

The method is limited by the sample size which, in this case, is the number of mean utility values. While the regression lines fitted to these seven observations showed a good correlation, the lack of data points limits the credibility of the method. In addition, the range of the values was narrow which in turn limits the range in which the method could be applied. However, the validation on external data proved that the equations for adjusting Dutch and German mean utilities worked within the range where they were developed. More data would also enable the exploration of which functional form yields the best adjustments over a wider range of mean values.

The present study did not include value sets which have been elicited using methods other than time trade-off. However, value sets elicited using other preference elicitation methods may also be used to calculate QALYs and equations for adjusting foreign mean utilities valued by value sets that applied other preference elicitation methods could be estimated in the same manner as the equations shown in this paper.

\section{Conclusion}

The present study showed that it is feasible to develop and validate regression models for adjusting mean utility estimates valued by foreign value sets that are systematically different from the national value set. The paper showed that adjustment has the potential to increase the transferability of a foreign utility to a national setting.

\section{Acknowledgments}

We are grateful to Sabrina Storgaard Sørensen and Flemming Witt Udsen for providing descriptive data on EQ-5D-3L answers. The study was funded by the North Denmark Region and Aalborg University which are public sector organizations. The funding sources were not involved in the process of designing, conducting, or reporting the work.

\section{Author contributions}

Both authors made substantial contributions to the data analysis, drafting, and critical revision of the manuscript, and approved for the final version to be published.

\section{Disclosure}

The authors report no conflicts of interest in this work.

\section{References}

1. Drummond MF, Sculpher MJ, Torrance GW, O'Brien BJ, Stoddart GL. Methods for the Economic Evaluation of Health Care Programmes. 3rd ed. New York: Oxford University Press; 2005.

2. National Institute for Health and Care Excellence (NICE). Guide to the Methods of Technology Appraisal. London; 2013.

3. Papaioannou D, Brazier J, Paisley S. Nice dsu technical support document 9: the identification, review and synthesis of health state utility values from the literature. University of Shefflield, Sheffield, 2011.

4. Knott R, Au N, Hollingsworth B, Lorgelly P. Anchoring vignettes and measures of health: adjusting for systematic differences in reporting behaviour. In: 10th World Congress in Health Economics for the International Health Economics Association (iHEA); July 12-16, 2014; Dublin, Ireland.

5. Bailey H, Kind P. Preliminary findings of an investigation into the relationship between national culture and EQ-5D value sets. Qual life Res. 2010;19(8):1145-1154.

6. Augestad LA, Rand-Hendriksen K, Kristiansen IS, Stavem K. Impact of transformation of negative values and regression models on differences between the UK and US EQ-5D time trade-off value sets. Pharmacoeconomics. 2012;30(12):1203-1214.

7. Knies S, Evers SMAA, Candel MJJM, Severens JL, Ament AJHA. Utilities of the EQ-5D: transferable or not? Pharmacoeconomics. 2009; 27(9):767-779.

8. Szende A, Oppe M, Devlin N. EQ-5D Value Sets: Inventory, Comparative Review and User Guide. The EuroQol Group; 2007; Dordrecht, the Netherlands.

9. Oppong R, Kaambwa B, Nuttall J, Hood K, Smith RD, Coast J. The impact of using different tariffs to value EQ-5D health state descriptions: an example from a study of acute cough/lower respiratory tract infections in seven countries. Eur J Heal Econ. 2013;14(2):197-209.

10. Badia X, Roset M, Herdman M, Kind P. A comparison of United Kingdom and spanish general population time trade-off values for eq-5d health states. Med Decis Mak. 2001;21(1):7-16.

11. Barbieri M, Drummond M, Rutten F, et al. What do international pharmacoeconomic guidelines say about economic data transferability? Value Health. 2010;13(8):1028-1037.

12. Welte R, Feenstra T, Jager H, Leidl R. A decision chart for assessing and improving the transferability of economic evaluation results between countries. Pharmacoeconomics. 2004;22(13):857-876.

13. Karlsson JA, Nilsson J-Å, Neovius M, et al. National EQ-5D tariffs and quality-adjusted life-year estimation: comparison of UK, US and Danish utilities in south Swedish rheumatoid arthritis patients. Ann Rheum Dis. 2011;70(12):2163-2166.

14. Parkin D, Rice N, Devlin N. Statistical analysis of EQ-5D profiles: does the use of value sets bias inference? Med Decis Mak. 2010; 30(5):556-565.

15. Chuang L, Zarate V, Kind P. Estimating domestic values for eq-5d health states using survey data from external sources. Med Care. 2009;47(2):168-175.

16. Dolan P. Modeling valuations for EuroQol health states. Med Care. 1997;35(11):1095-1108.

17. Lamers LM, Mcdonnell J, Stalmeier PFM, Krabbe PFM, Busschbach JJV. The Dutch tariff: results and arguments for an effective design for national EQ-5D valuation studies. Health Econ. 2006;15:1121-1132.

18. Greiner W, Claes C, Busschbach JJV. Schulenburg J-M. Validating the EQ-5D with time trade off for the German population. Eur J Heal Econ. 2005;6(2):124-130.

19. Oddershede L, Andreasen JJ, Ehlers L. Estimation of utility values from visual analog scale measures of health in patients undergoing cardiac surgery. Clin Outcomes Res. 2014;6(1):21-27.

20. Oddershede L, Walker S, Paton N, Stöhr W, Dunn D, Sculpher MJ. Cost-effectiveness analysis of protease inhibitor monotherapy vs ongoing triple-therapy in the long-term management of HIV patients. Value Health. 2014;17(7):A683.

21. Udsen FW, Lilholt PH, Hejlesen O, Ehlers LH. Effectiveness and cost-effectiveness of telehealthcare for chronic obstructive pulmonary disease: study protocol for a cluster randomized controlled trial. Trials. 2014; 15:178. 
22. Petersen KD, Kronborg C, Gyrd-Hansen D, Dahl R, Larsen JN, Linneberg A. Characteristics of patients receiving allergy vaccination: to which extent do socio-economic factors play a role? Eur J Public Health. 2011;21(3):323-328.

23. Hvidberg MF, Brinth LS, Olesen AV, Petersen KD, Ehlers L. The health-related quality of life for patients with myalgic encephalomyelitis/chronic fatigue syndrome (ME/CFS). PLoS One. 2015;10(7):e0132421.
24. Sørensen SS, Pedersen KM, Weinreich UM, Ehlers LH. Design, and participant enrollment, of a randomized controlled trial evaluating effectiveness and cost-effectiveness of a community-based case management intervention, for patients suffering from COPD. Open Access J Clin Trials. 2015;7:53-62.

\section{Publish your work in this journal}

ClinicoEconomics \& Outcomes Research is an international, peerreviewed open-access journal focusing on Health Technology Assessment, Pharmacoeconomics and Outcomes Research in the areas of diagnosis, medical devices, and clinical, surgical and pharmacological intervention. The economic impact of health policy and health systems organization also constitute important areas of coverage. The manuscript management system is completely online and includes a very quick and fair peer-review system, which is all easy to use. Visit http://www.dovepress.com/testimonials.php to read real quotes from published authors.

Submit your manuscript here: http://www.dovepress.com/clinicoeconomics-and-outcomes-research-journal 